Metastatic carcinomas in hernial sacs are rare, especially in bilateral femoral hernias. Here we describe a 63-yearold female patient with metastatic carcinoma in bilateral femoral hernias. She was diagnosed incidentally during femoral hernioplasty. A diagnostic laparoscopy revealed metastatic nodules in the abdominal cavity. Subsequently, the patient underwent a laparotomy that did not show any evidence of primary lesions. A chemotherapeutic pump was implanted into the abdominal cavity during surgery for postoperative chemotherapy. The chemotherapy regimen included 5-Fu, hydroxycamptothecin, and pirarubicin, supplemented with high agglomerative staphylococcin. Forty-one months after surgery her disease was stable and no abdominal cavity, pelvis, liver or lung metastases were observed. Therefore, we suggest that abnormal hernial sacs may be the first clue to an underlying cancer, and should be sent for histological examination. Furthermore, if a patient is found to have metastatic abdominal cancer with no evidence of primary lesions, they would probably benefit from postoperative chemotherapy. This can be delivered using a chemotherapeutic pump implanted during surgery.

Key words: metastatic carcinoma, malignant abdominal tumour, bilateral femoral hernioplasty, primary lesions.

Contemp Oncol (Pozn) 2014; 18 (2): 130-133 DOI: $10.5114 /$ wo.2014.42728

\section{Incidental finding of a malignant tumour in an inguinal hernia sac}

\author{
Rong Qin, Qiaoyu Zhang, Jianfeng Weng, Yongdong Pu
}

Department of General Surgery, The $309^{\text {th }}$ Hospital of PLA, Beijing, PR China Dr. Rong Qin and Qiaoyu Zhang contributed equally and are co-first authors.

\section{Introduction}

Malignant tumours in the regions of the gastrointestinal tract, biliary tract, pancreas, appendix and adnexa may metastasise to the peritoneum and greater omentum through implantation, causing ascites. The primary sites of most tumours can be definitely diagnosed using computed tomography $(\mathrm{CT})$, endoscopy or exploratory laparotomy. In patients with extra-abdominal hernia-complicating peritoneal metastatic carcinoma, the cancer cells can be implanted in the hernia sacs. However, metastatic carcinomas in hernial sacs are rare, and most previous cases had a pathologically proven primary tumour site [1]. Metastatic carcinomas in bilateral femoral hernias are extraordinarily rare. Here we report the incidental diagnosis of a malignant abdominal tumour during repair of bilateral femoral hernias. In our case, the primary tumour was not found, even after diagnostic laparoscopy and laparotomy. Forty-one months after surgery the patient had a stable disease.

\section{Case report}

A 63-year-old female patient was referred to our hospital with bilateral groin lumps. Her medical history was significant for cholecystitis, status post cholecystectomy, approximately 8 years prior. One month before admission to our hospital, she received treatment at another hospital for ascitic fluid of unknown aetiology. Physical examination revealed normally development, eutrophy and abdominal uplift. Abdominal palpation showed no liver or spleen enlargement and no abdominal lump, but it showed bilateral groin lumps that were $3 \mathrm{~cm} \times 3 \mathrm{~cm} \times 3 \mathrm{~cm}$, and $2 \mathrm{~cm} \times 2 \mathrm{~cm} \times 2 \mathrm{~cm}$. The lumps were clearly demarcated, with a smooth surface, moderate texture and poor mobility. Ultrasounds of the liver, pancreas, spleen, uterus and adnexa were all normal. In addition, ultrasound suggested that the bilateral groin lumps extended into the abdominal cavity (Fig. 1). Under epidural anaesthesia, tension-free hernioplasty was performed to repair the bilateral femoral hernias that presented as groin lumps. The wall of the left femoral hernia sac showed thickening, and the contents of the lump appeared to be necrotic. The right femoral hernia sac contained fluid, and the wall showed uneven thickening. The resected tissue and hernia sacs underwent pathological examination. The hernia sacs contained metastatic adenocarcinoma. Histologically, the tumour appeared to originate from breast or ovarian tissue. Therefore, molybdenum target mammography and other related tumour marker evaluations were performed. However, molybdenum target mammography was normal, and tumour markers were within normal limits: $\alpha$-fetoprotein $($ AFP $)=2.3 \mathrm{ng} / \mathrm{ml}$; carcinoembryonic antigen $(C E A)=1.5 \mathrm{ng} / \mathrm{ml}$; carbohyd rate antigen $50($ CA 50) $<5 \mu / \mathrm{ml}$; carbohydrate antigen $125($ CA125) $=35.3$ $\mu / \mathrm{m}$; carbohydrate antigen 19-9 (CA19-9) $=1.1 \mu / \mathrm{ml}$; and carbohydrate antigen 15-3 $($ CA15-3) $=15.1 \mu / \mathrm{ml})$. Thus, there was no evidence of a primary tumour. In order to further explore the presence of a primary tumour, laparotomy was performed under general anaesthesia two weeks later, during 

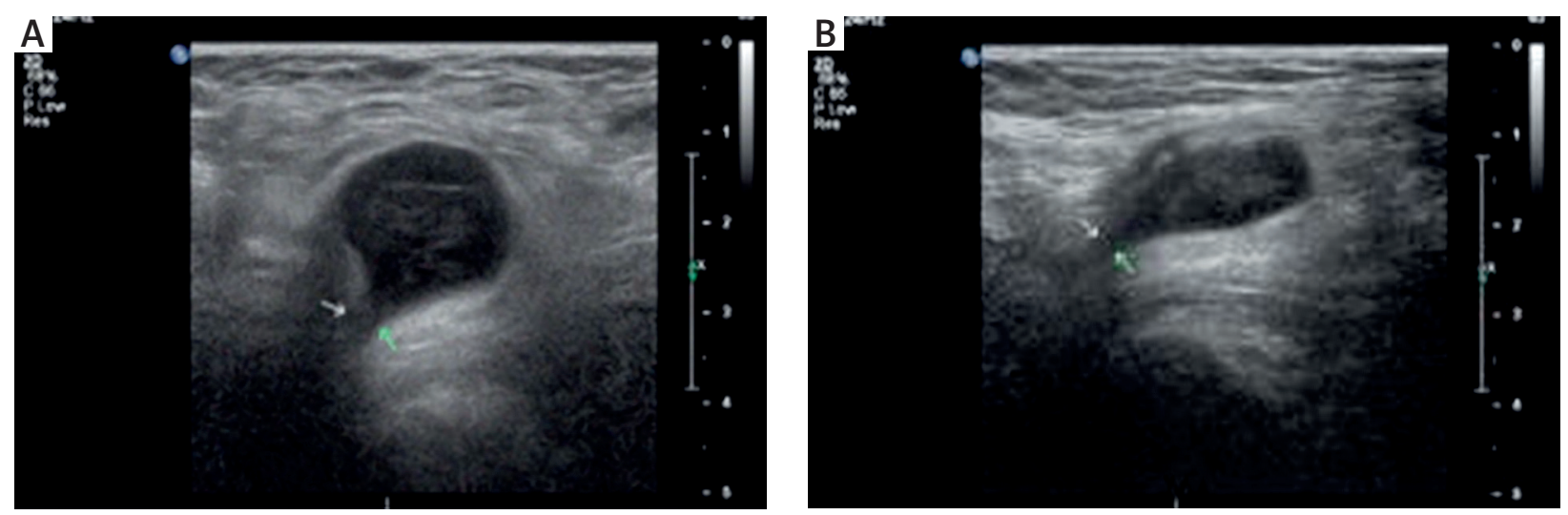

Fig. 1. Ultrasound images of the bilateral femoral hernias: A) left femoral hernia; B) right femoral hernia

which light-yellow transparent fluid was observed in the abdominal cavity. During the surgery, nodules of the same size as mung beans (about $0.7 \mathrm{~cm} \times 0.6 \mathrm{~cm} \times 0.6 \mathrm{~cm}$ ) were observed in the left peritoneum. White miliary nodules (about $0.2 \mathrm{~cm} \times 0.2 \mathrm{~cm} \times 0.2 \mathrm{~cm}$ ) were observed at the bottom of the pelvic cavity, which involved the left mesosalpinx. The greater omentum was massive, with a size of $8 \mathrm{~cm} \times 6 \mathrm{~cm} \times 3 \mathrm{~cm}$. There were no pathological changes in other organs including the ileocecal junction and appendix. The mung bean-sized nodules within the left peritoneum were resected, while the miliary nodules in the pelvic floor region were treated using electrosurgical cautery. The greater omentum and bilateral adnexa were resected and underwent immunohistochemical analysis, while the appendix was not removed because it was normal. In order to give postoperative chemotherapy, an abdominal chemotherapeutic pump was implanted. The results of the postoperative histopathology report and immunohistochemical analysis were as follows:

The mesosalpinx lymph nodes and greater omentum nodules were observed with poorly differentiated adenocarcinoma metastasis. The membranous epithelial marker cytokeratin $(\mathrm{CK}, \mathrm{AE} 1)(+++)$, breast carcinoma related antigen CA15-3 (++), mesothelium and other sources of markers were all shown to be negative. No carcinoma was observed at the bilateral ovaries or fallopian tubes. Part of the greater omentum region exhibited panniculitis changes. (CK, AE1) (+++), (CK, AE3) (+), CK7 (-), CK2O (-), epithelial specific antigen (ESA) (++), mesothelial (-), calretinin (-), carcinoembryonic antigen (CEA) $(-)$, HEPATOCYTE $(-)$, CA125 (+/-), CA19-9 (-), leukocyte common antigen (LCA) (-), CD45RO (-), cluster of differentiation 20 (CD20) $(-)$, S-100 (-), neuron-specific enolase (NSE) $(-)$, thyroid transcription factor (TTF) (-), human melanoma, black-45 (HMB45) (-), CD34 (-), Vimentin (VIM) (-), CA15-3 (++), presenilin 2 (PS2) (-). The greater omentum lymph nodes and left mesosalpinx were pathologically proven to have poorly differentiated adenocarcinoma (Fig. 2A, B). Immunohistochemical analysis of CK (AE1) and CA15-3 showed moderate positivity (Fig. 2C,D). Markers of other organs and mesothelial tissues were all negative. Tumour cells were not observed in the ovaries or uterine tubes. Most areas of the greater omentum showed changes consistent with panniculitis. A month-long postoperative chemotherapy treatment, which consisted of four courses, was carried out two weeks later. During the course, the patient received $0.5 \mathrm{~g}$ once daily of 5 -Fu from day 1 to day 5 , using the abdominal chemotherapeutic pump that was implanted during surgery. Twenty milligrams hydroxycamptothecin, and $20 \mathrm{mg}$ pirarubicin were given by intravenous drip on days 2, 4, 6 and 8. Forty-one months after surgery, a computed tomography (CT) found no lesions in the liver, pancreas, spleen, biliary tract, lung, kidneys, ileocecal junction and appendix. There was also no evidence of a pleural effusion, ascites or enlargement of lymph nodes in the abdominal cavity and groin. Breast and uterine ultrasounds were normal. The expression levels of tumour markers were within normal limits (AFP: $2.1 \mathrm{ng} / \mathrm{ml}$, CEA: $2.2 \mathrm{ng} / \mathrm{ml}$, CA50: $2.2 \mu / \mathrm{ml}$, CA125: $8.45 \mu / \mathrm{ml}$, CA19-9: 14.67 $\mu / \mathrm{ml}$, CA15-3: $9.38 \mu / \mathrm{ml})$.

\section{Discussion}

Here we report an elderly female patient with metastatic carcinoma inside bilateral femoral hernial sacs. Her cancer was diagnosed incidentally during hernia repair, and the metastatic tumour was pathologically proven to be adenocarcinoma. Hernia is a common surgical disease, and cases of metastatic carcinoma in inguinal hernias have occasionally been reported [2-4]. However, it is extraordinarily rare to find bilateral metastatic carcinoma in right and left femoral hernias. In our case, the patient had a 2-year history of slow growing lumps in her groin. The formation of metastatic carcinoma in femoral hernias may be related to a few factors. First, a growing primary tumour may directly extend to the peritoneal viscera of hernia sacs. Second, it is possible that shedding tumour cells traveled immersed in ascites to the hernia sacs.

In our case, the primary tumour was not identified even after diagnostic laparoscopy and laparotomy. Malignant peritoneal mesothelioma has frequently been observed to metastasise to the peritoneum [5]. The incidence of this neoplasm is $2-2.6$ cases per million annually $[6,7]$, and the prognosis is very poor. Therefore, it is unlikely that peritoneal mesothelioma is the cause of our patients' cancer. Other cancers, such as ovarian, appendix and pancreatic, have also been shown to metastasise to the peritoneum 

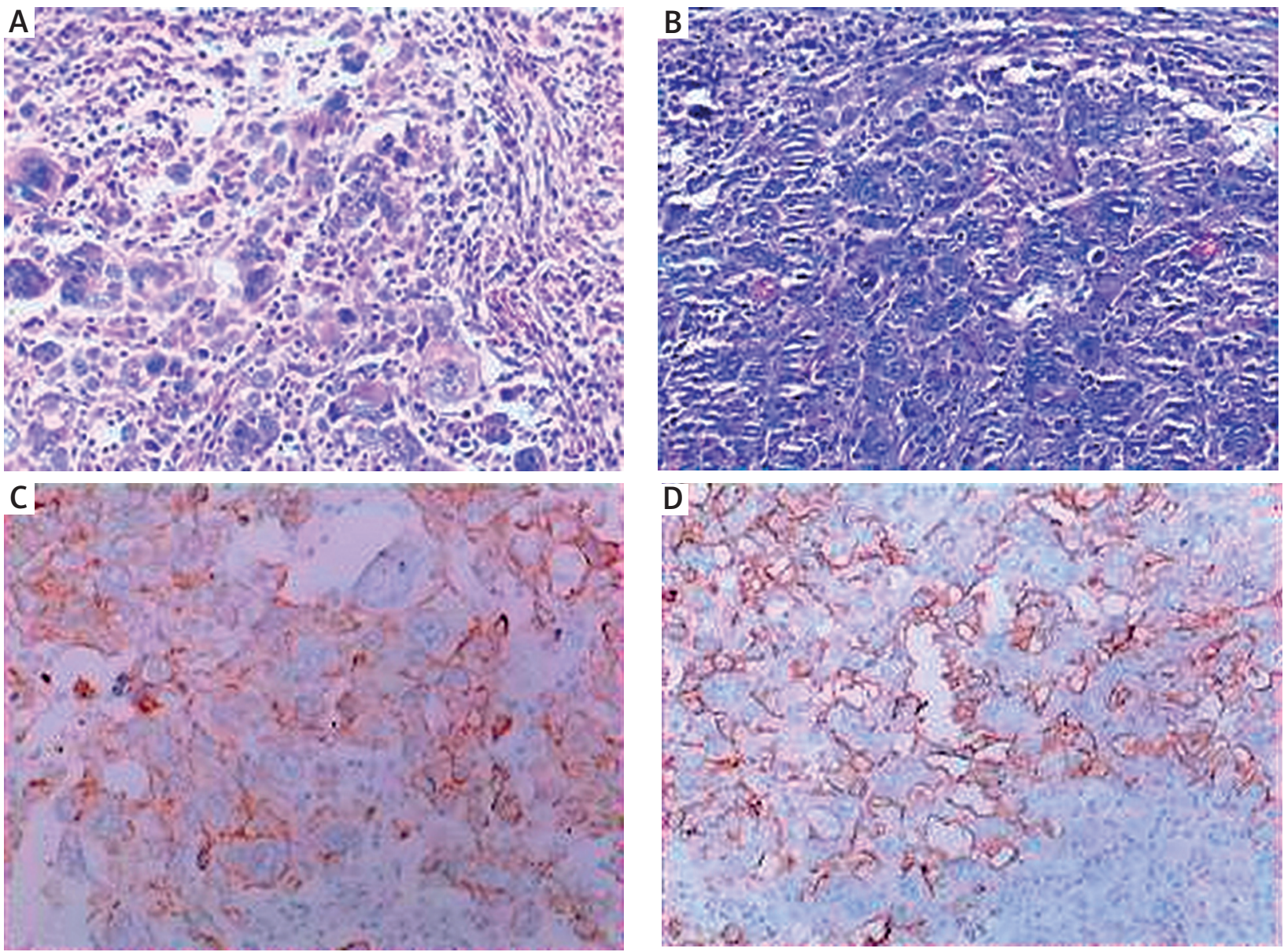

Fig. 2. Histological haematoxylin-eosin (HE) and immunohistochemical analysis of the nodules (100x): A) HE analysis of the left mesosalpinx metastasis; B) HE analysis of the greater omentum lymph node metastases; C) Immunohistochemical analysis of CK (AE1); D) Immunohistochemical analysis of CA15-3

$[8,9]$. The pathologic and radiographic data in our case demonstrated no lesions or mucinous-like appearance in the ovaries, appendix or pancreas. In addition, the ascites was clear and transparent, excluding these cancer types. Molybdenum target mammography showed no breast abnormalities, and breast cancer tumour markers were within normal limits, excluding breast cancer. The left mesosalpinx was pathologically proven to contain malignant cells. However, these malignant cells were localised in the mesosalpinx and no primary lesion was found in the ovaries, so these were considered to be metastatic lesions. Thus, the possibility of ovarian cancer was also excluded. The patient was subjected to cholecystectomy eight years prior, and neither the intraoperative exploration nor a postoperative follow-up showed abnormalities in the biliary tract, which suggested that the tumour did not originate from the biliary system. Preoperative gastroscopy and colonoscopy were performed and did not show evidence of lesions in the digestive tract. In addition, stool occult blood was negative.

The patient received postoperative chemotherapy that included 5-Fu, hydroxycamptothecin and pirarubicin, supplemented with high agglomerative staphylococcin. 5-Fu was given using an abdominal chemotherapeutic pump; hydroxycamptothecin, pirarubicin and high agglomerative staphylococcin were given by intravenous drip. Forty-one months after surgery the patient had a stable disease, with no evidence of pelvic cavity, appendix, liver or lung metastases.

In conclusion, we suggest that abnormal hernial sacs may be the first clue to an underlying cancer, and they should be sent for histological examination. Furthermore, if a patient is found to have metastatic abdominal cancer with no evidence of primary lesions, they would probably benefit from postoperative chemotherapy after the resection or cauterisation of these lesions. This can be delivered using a chemotherapeutic pump implanted during surgery.

Authors declare no conflict of interests.

This work was supported by the Third Military Medical University Trauma, Burn and Combined Injury, National Key Laboratory Open Foundation in 2009 (SKLKF 200911).

\section{References}

1. Nicholson CP, Donohue JH, Thompson GB, Lewis JE. A study of metastatic cancer found during inguinal hernia repair. Cancer 1992; 69: 3008-11

2. Chen KT. Metastatic carcinoma in inguinal hernia sac. J Surg Oncol $1984 \cdot 25 \cdot 248-9$ 
3. Goyal A, Mansel RE, Goyal S. Gastrointestinal stromal tumour in an inguinal hernial sac: an unusual presentation. Postgrad Med J 2003; 79: 707-8.

4. Patel T, Viswanathan S, Jambhekar NA. Metastatic adenocarcinoma presenting as an inguinal hernia: a case report and review of literature. Indian J Pathol Microbiol 2007; 50: 541-2.

5. Chatzipantelis P, Kairi-Vassilatoul E, Smyrniotis V, Pafiti A. Incidental finding of a malignant peritoneal mesothelioma in a inguinal hernia sac: report of a case. Eur J Gynaecol Oncol 2006; 27: 534-6.

6. Graia S, Scafa F, Baiardi P, Candura SM. Malignant mesothelioma: analysis of a hospital case series. G Ital Med Lav Ergon 2012; 34 (3 Suppl): 558-60

7. Berk S, Dogan OT, Kilickap S, Epozturk K, Akkurt I, Seyfikli Z. Clinical characteristics, treatment and survival outcomes in malignant mesothelioma: eighteen years' experience in Turkey. Asian Pac J Cancer Prev 2012; 13: 5735-9.

8. Marchal N, Gennigens C, Jerusalem G. Peritoneal carcinomatosis of unknown origin. Rev Med Liege 2012; 67: 582-6.

9. Pisano G, Erdas E, Licheri S, Dazzi C, Pomata M, Daniele GM. Uncommon pathologies discovered during exploratory laparoscopy for malignant diseases. Chir Ital 2007; 59: 793-9.

\section{Address for correspondence}

\section{Yongdong $\mathrm{Pu}$}

Department of General Surgery

The $309^{\text {th }}$ Hospital of PLA

No. 17, Heishanhu Road

Beijing 100091, China

tel. +86-10-66775914

e-mail: yongdongpu@126.com

Submitted: 16.07 .2013

Accepted: 8.06 .2014 